\title{
Realtime Implementation of Visual-aided Inertial Navigation Using Epipolar Constraints
}

\author{
John-Olof Nilsson, Dave Zachariah, Magnus Jansson, and Peter Händel \\ Signal Processing Lab, ACCESS Linnaeus Centre, KTH Royal Institute of Technology, Stockholm, Sweden
}

\begin{abstract}
A real-time implementation and the related theory of a visual-aided inertial navigation system are presented. The entire system runs on a standard laptop with off-the-shelf sensory equipment connected via standard interfaces. The visual-aiding is based on epipolar constraints derived from a finite visual memory. The navigational states are estimated with a squareroot sigma-point Kalman filter. An adaptive visual memory based on statistical coupling is presented and used to store and discard images selectively. Timing and temporal ordering of sensory data are estimated recursively. The computational cost and complexity of the system is described, and the implementation is discussed in terms of code structure, external libraries, and important parameters. Finally, limited performance evaluation results of the system are presented.
\end{abstract}

\section{INTRODUCTION}

A monocular camera and inertial sensors are an attractive sensor combination for autonomous positioning and navigation: the setup is intuitive in that it resembles our own senses; the sensor hardware is inexpensive, compact, easily available, and can be found in many consumer products; it is independent of external infrastructure; and the inertial sensors are selfcontained and have a high integrity and dynamic range, while the camera imagery contains an excess of information. Several methods capable of stand-alone positioning based on this sensor combination can be found in the literature, e.g. [1][2][3]. However, there are few presentations of full realtime, online implementations and related system analysis, see [4] for an exception. Realtime capabilities are often measured in terms of computational time or assessed by partial system analysis. A complete implementation would uncover many theoretical and practical issues that need to be resolved for a realtime system to work online. It would also validate the theoretical claims of the system analysis. The step from an algorithm to a realtime implementation might seem deceptively short when described on paper but in reality, it can often be quite long and cumbersome.

Therefore, in this article we present a realtime implementation and related theory and analysis of a monocular visual-aided inertial navigation system (INS). The extraction of visual information is based on comparisons between the current camera image with a set of images in a finite visual memory. Epipoles are calculated and used as observations related to the navigational states. The basic visual information extraction method has previously been presented and used on synthetic data in [5]. A similar approach has also been presented in [6]. Figure 1 shows an illustration of the system components and functional blocks. A concise description of

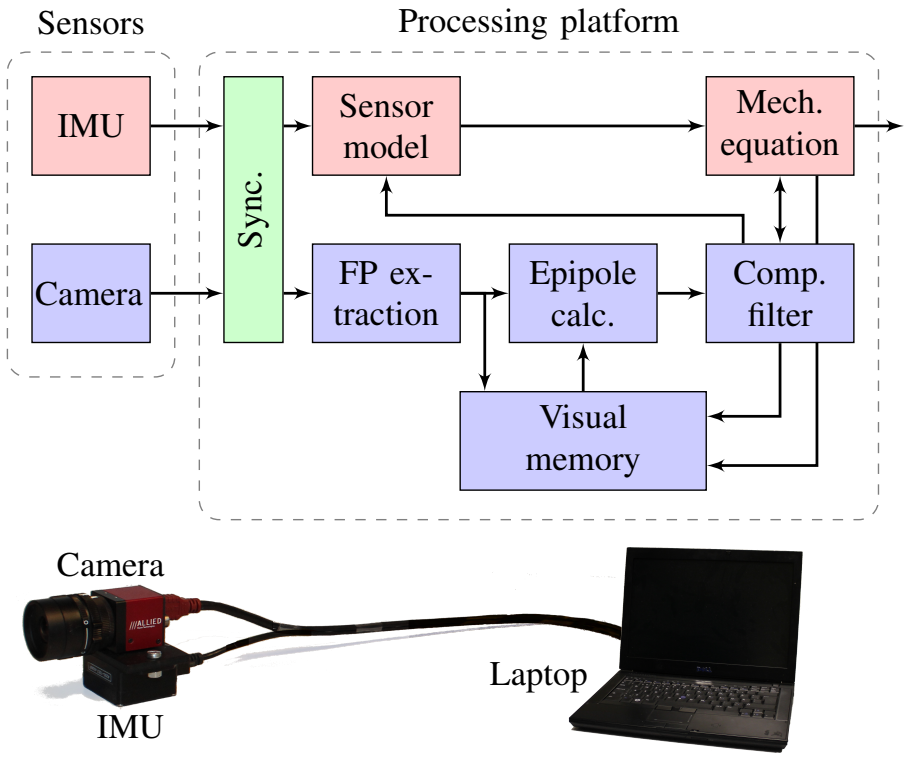

Fig. 1. Illustration of processing and hardware of the implementation of the vision-aided INS. The red blocks constitute the INS while the blue blocks give the visual-aiding. The green block handles timing (synchronization) in the system. For the processing platform, a standard laptop has been used. Note that sensors units and laptop are not to scale.

the theory behind the visual-aiding is given and the related filtering is implemented with a sigma-point Kalman filter in a numerically robust square-root form, which we have found necessary for real-data processing. For ease of implementation, the visual information extraction method is presented in a setbased framework using homogeneous coordinates. To limit the visual memory, and thereby the computational cost, a novel adaptive memory has been developed, which uses statistical coupling to determine which past views to preserve and which to discard. This memory has potential bearing on all methods that use a finite memory to generate observations. The system hardware implementation is based on off-the-shelf components and therefore no hardware synchronization of sensory data is assumed. Time synchronization and temporal ordering are based on methods previously presented in [7] and are briefly described here. A description of the implementation in terms of code structure, external libraries, and hardware is given. For the whole system realtime capability on a standard laptop is achieved. Unlike in [4], no external processing hardware, such as graphical processing units, are used. An analysis and measurement of the computational costs and processing 
complexity and related trade-offs in parameter settings are presented. Finally, limited performance evaluation tests are presented and conclusions drawn.

\section{INERTIAL NAVIGATION}

The inertial navigation is the backbone of the presented visual-aided INS implementation. The INS propagates and outputs the main navigational states of the system: position $\mathbf{p} \in \mathbb{R}^{3 \times 1}$, velocity $\mathbf{v} \in \mathbb{R}^{3 \times 1}$, and orientation $\mathbf{q} \in \mathbb{H}$. The position $\mathbf{p}$ and velocity $\mathbf{v}$ are in the navigation coordinate frame $n$, which is the local tangent plane, and the orientation $\mathbf{q}$ is the unit quaternion describing the rotation between the $n$ frame and the body coordinate frame $b$. The $b$ frame is identical to the sensor frame of the inertial measurement unit (IMU).

The mechanization equations for the INS are of first-order discretization type, i.e.

$$
\begin{aligned}
\mathbf{p}_{k+1} & =\mathbf{p}_{k}+\mathbf{v}_{k} d t_{k} \\
\mathbf{v}_{k+1} & =\mathbf{v}_{k}+\left(\mathbf{q}_{k} \mathbf{f}_{k} \mathbf{q}_{k}^{-1}-\mathbf{g}\right) d t_{k} \\
\mathbf{q}_{k+1} & =\boldsymbol{\Omega}\left(\boldsymbol{\omega}_{k} d t_{k}\right) \mathbf{q}_{k},
\end{aligned}
$$

where the Coriolis term has been neglected; $k$ is a time index and $d t_{k}$ is the time difference between time instants with indices $k+1$ and $k ; \mathbf{g}=\left[\begin{array}{lll}0 & 0 & g\end{array}\right]^{\mathrm{T}}$ is the gravity vector where $g$ is the local gravitational field strength; $\mathbf{f} \in \mathbb{R}^{3 \times 1}$ is the specific force and $\boldsymbol{\omega} \in \mathbb{R}^{3 \times 1}$ are the rotational rates in $b$; and $\Omega(\cdot)$ is the quaternion update matrix. For a detailed treatment of inertial navigation, see [8]. For a discussion of how to get the time indices $k$ and differentials $d t_{k}$, see Section VII.

The IMU provides measurements of the specific force $\tilde{\mathbf{f}}_{k}$ and of the rotational rates $\tilde{\boldsymbol{\omega}}_{k}$. Compounding the inertial measurement into a measurement vector $\tilde{\mathbf{u}}_{k}=\left[\begin{array}{ll}\tilde{\mathbf{f}}_{k}^{\mathrm{T}} & \tilde{\boldsymbol{\omega}}_{k}^{\mathrm{T}}\end{array}\right]^{\mathrm{T}}$ and the specific force and the angular rates into an input vector $\mathbf{u}_{k}=\left[\begin{array}{ll}\mathbf{f}_{k}^{\mathrm{T}} & \boldsymbol{\omega}_{k}^{\mathrm{T}}\end{array}\right]^{\mathrm{T}}$, the inertial measurements and the measurement errors are modeled as

$$
\tilde{\mathbf{u}}_{k}=\mathbf{u}_{k}+\mathbf{b}_{k}+\mathbf{w}_{k}^{u} \quad \text { and } \quad \mathbf{b}_{k+1}=\mathbf{b}_{k}+d t_{k} \mathbf{w}_{k}^{b},
$$

where $\mathbf{w}_{k}^{u}$ is white measurement noise, $\mathbf{b}_{k}$ is slow varying sensor biases, and $\mathbf{w}_{k}^{b}$ is a white driving noise terms.

Combining the INS states into a state vector $\mathbf{x}_{k}^{\text {ins }}=$ $\left[\begin{array}{lll}\mathbf{p}_{k}^{\mathrm{T}} & \mathbf{v}_{k}^{\mathrm{T}} & \mathbf{q}_{k}^{\mathrm{T}}\end{array}\right]^{\mathrm{T}}$, relation (1) can compactly be written as a function $\mathbf{x}_{k}^{\text {ins }}=f_{i m u}\left(\mathbf{x}_{k-1}^{\text {ins }}, \mathbf{u}_{k}\right)$. The inertial navigation alone is deterministic. Even though the sensor errors are stochastic, without aiding, there is no further information to estimate them. Consequently, given some initial state estimates $\hat{\mathbf{x}}_{0}$ and bias estimates $\hat{\mathbf{b}}_{0}$, the estimates can be propagated in time by

$$
\begin{aligned}
& \hat{\mathbf{x}}_{k+1}^{\text {ins }}=f_{\text {ins }}\left(\hat{\mathbf{x}}_{k}^{\text {ins }}, \tilde{\mathbf{u}}_{k}-\hat{\mathbf{b}}_{k}\right) \\
& \hat{\mathbf{b}}_{k+1}=\hat{\mathbf{b}}_{k}
\end{aligned}
$$

for all $k>0$. This way the INS works as the system backbone, continuously providing state estimates with the rest of the system having the role of correcting it.

\section{VISUAL INFORMATION}

The visual information extraction is the process of extracting spatial information from the imagery. Since the imagery is invariant under scene scaling, it cannot give absolute spatial relations and therefore we refer to the spatial information as visual constraints. Further, since the images are a result of overlapping scenes, we refer to them as views.

All views could jointly be used to calculate the visual constraints. However, for realtime navigation, the information from a new image needs to be incorporated recursively. Therefore, for each time instant we only consider the pairwise relations between the most recent view and past views.

\section{A. Feature points}

The views contain an excess of information and therefore, they must be interpreted and condensed somehow. The most commonly used method for this is to establish point correspondences between views. For this, we use feature points (FPs) $\mathbf{z}_{p}^{(i)} \in \mathbb{R}^{3 \times 1}$ indexed by $p$ and selected in the view (i) based on some distinctiveness measure. This way the visual information of a view is condensed into few distinct and well-localized quality points. The FPs are in homogenous coordinates, meaning that a point $\left[\begin{array}{ll}x & y\end{array}\right]$ in the image plane is represented as $\left[\begin{array}{lll}x & y & 1\end{array}\right]$. The FPs are accompanied by descriptors $\boldsymbol{\eta}_{p}^{(i)}$ that are vectors describing the points. Define the set of all interest points $\mathcal{Z}^{(i)}=\left\{\mathbf{z}_{p}^{(i)}\right\}$ and the set of all descriptors $\mathcal{N}^{(i)}=\left\{\boldsymbol{\eta}_{p}^{(i)}\right\}$ from view $(i)$. Based on these, the set of all point correspondences, between the current view $(c)$ and the past view $(i)$, are consolidated by

$$
\mathcal{M}^{(i)}=\left\{\{p, q\}:\left\|\boldsymbol{\eta}_{p}^{(c)}-\boldsymbol{\eta}_{q}^{(i)}\right\|_{\eta}<\gamma_{\eta}\right\}
$$

where $\gamma_{\eta}$ is some similarity threshold. The set $\mathcal{M}^{(i)}$ constitutes the basic spatial information between views.

\section{B. Visual constraints}

Geometrically the point correspondences between the current view $(c)$ and view $(i)$ are governed by epipolar geometry and the fundamental matrix $\mathbf{F}^{(i)} \simeq \mathbf{A}^{-T}\left[\mathbf{p}^{(c)}-\right.$ $\left.\mathbf{p}^{(i)}\right]_{\times} \mathbf{R}_{(i)}^{(c)} \mathbf{A}^{-1}$ where $\simeq$ denotes similarity up to scale; $\mathbf{A}$ is the camera calibration matrix; $[\cdot]_{\times}$is the cross-product matrix; $\mathbf{p}^{(c)}$ and $\mathbf{p}^{(i)}$ are the positions of the focal points of view $(i)$ and $(c)$; and $\mathbf{R}_{(i)}^{(c)}$ is the rotation matrix between the views. In terms of the navigation states, the focal point is

$$
\mathbf{p}^{(i)}=\mathbf{p}_{\ell}+\mathbf{q}_{\ell} \mathbf{p}_{(c)}^{b} \mathbf{q}_{\ell}^{-1}
$$

where $\ell$ is the time instant the image of view $(i)$ was taken and $\mathbf{p}_{(c)}^{b}$ is the displacement between the $b$ frame and the camera focal point in the $b$ frame. Similarly, the rotation matrix is

$$
\mathbf{R}_{(i)}^{(c)}=g_{\mathrm{R}}\left(\mathbf{d}^{-1} \mathbf{q}_{k}^{(i)}{ }^{-1} \mathbf{d}\right)
$$

where $\mathbf{d}$ is the static rotation between the camera coordinate frame of view $(c)$ and the $b$ frame, $\mathbf{q}_{k}^{(i)}$ is the rotation between the coordinate frame of view $(i)$ and the $b$ frame, and $g_{\mathrm{R}}$ is the mapping between quaternions and rotation matrices. 
Ideally, under perfect FP detection and matching, the point correspondences and the fundamental matrix are related by

$$
\mathbf{z}_{p}^{(c)}{ }^{\mathrm{T}} \mathbf{F}^{(i)} \mathbf{z}_{q}^{(i)}=0:\{p, q\} \in \mathcal{M}^{(i)} .
$$

The fundamental matrix describes the spatial relation between different views completely[9]. Therefore, relation (6) will be the foundation for all visual constraints.

The visual constraint can be calculated for all past images. However, this gives a growing computational cost, which is clearly unacceptable. For many situations, the navigation system will be in motion such that within a short period of time, there is no overlapping perspective between views and consequently no matches and no visual constraints. To remedy this, a visual memory of finite size $P$ is used

$$
\mathcal{V}=\left\{\left\{\mathcal{Z}^{(1)}, \mathcal{N}^{(1)}, \mathbf{p}^{(1)}, \mathbf{q}_{k}^{(1)}\right\}, \cdots,\left\{\mathcal{Z}^{(P)}, \mathcal{N}^{(P)}, \mathbf{p}^{(P)}, \mathbf{q}_{k}^{(P)}\right\}\right\} .
$$

For further details of how the views are inserted and removed from the memory see Section VI.

\section{Epipole constraints}

The problem with (6) is that it cannot easily be posed as an observation related to the navigational states. Further, estimating $\mathbf{F}^{(i)}$ from $\mathcal{M}^{(i)}$ and $\mathcal{Z}^{(i)}$ is computationally demanding and establishing a statistical description of the errors is difficult. Instead we have previously demonstrated that visual constraints from the matches can be extracted by unwrapping the rotation of the features points by the orientation estimates of the inertial navigation and calculating epipoles from the unwrapped matches [5].

The idea is that under the pure translational motion, the fundamental matrix becomes $\mathbf{F}^{(i)} \simeq \mathbf{A}^{-T}\left[\mathbf{p}^{(c)}-\mathbf{p}^{(i)}\right]_{\times} \mathbf{A}^{-1}$. Then the null-space of $\mathbf{F}^{(i)}$ is described by the epipole $\mathbf{y}^{(i)}$ of the $(i)$ th view relative to the current view, i.e. $\mathbf{F}^{(i)} \mathbf{y}^{(i)}=\mathbf{0}$. Define the mapping $g_{r}:\left[\begin{array}{lll}x & y & z\end{array}\right]^{\mathrm{T}} \mapsto\left[\begin{array}{ll}x / z & y / z\end{array}\right]$. Then, this means that

$$
\mathbf{y}^{(i)}=\overline{\mathbf{A}} g_{r}\left(\Delta \mathbf{p}^{(i)}\right) \text {. }
$$

where $\Delta \mathbf{p}^{(i)}=\mathbf{d} \mathbf{q}_{k}\left(\mathbf{p}^{(c)}-\mathbf{p}^{(i)}\right) \mathbf{q}_{k}{ }^{-1} \mathbf{d}^{-1}$ is the relative translation between views in the current view frame and $\overline{\mathbf{A}}=\mathbf{A}_{1: 2,1: 3}$ is the truncated camera calibration matrix. In contrast to (6), the epipole visual constraint (7) is, via (4), directly amenable as an observation related to the navigational states and is the constraint used in the current system.

\section{Epipole measurement}

For (7) to hold, pure translation between views is required; but this will not hold in the general case. However, the inertial navigation provides accurate $\left(\sim 1^{\circ}\right)$ relative orientation information over time periods up to minutes. Combined with the visual-aiding itself, it can be expected that the relative orientation information over the maximum time baseline in the finite visual memory is good and relative orientation estimates can be used to create apparent pure translation, i.e. parallel image planes. This is achieved by correcting the FPs for the relative orientation of views. We refer to this procedure as rotation unwrapping.
To unwrap the rotation of the past views, the relative rotation between the current view and the past views is needed. Therefore, the orientations of views $\mathbf{q}_{k}^{(i)}$ in $\mathcal{V}$ relative to the current view are kept in a matrix $\boldsymbol{\Theta}_{k}=\left[\mathbf{q}_{k}^{(1)} \cdots \mathbf{q}_{k}^{(P)}\right]$. As soon as the orientation $\mathbf{q}_{k}$ in $\mathbf{x}_{k}^{\text {ins }}$ is updated, the matrix $\boldsymbol{\Theta}_{k}$ is updated by

$$
\boldsymbol{\Theta}_{k+1}=\boldsymbol{\Omega}\left(\boldsymbol{\omega}_{k} d t_{k}\right) \boldsymbol{\Theta}_{k}
$$

When the view $(c)$ is inserted in $\mathcal{V}$ at time instant $k$ as a view $(i)$, the corresponding orientation is set to $\mathbf{q}_{k}^{(i)}=$ $\left[\begin{array}{llll}0 & 0 & 0 & 1\end{array}\right]$.

The rotations of view $(i)$ are unwrapped by correcting the FPs in $\mathcal{Z}^{(i)}$ by the rotation $\mathbf{q}_{k}^{(i)}$. In homogenous coordinates this is done simply by a vector rotation followed by a renormalization. Define the mapping $g_{1 / z}:\left[\begin{array}{lll}x & y & z\end{array}\right]^{\mathrm{T}} \mapsto$ $\left[\begin{array}{llll}x / z & y / z & 1\end{array}\right]^{\mathrm{T}}$. Then a FP $\mathbf{z}_{p}^{(i)}$ is corrected for the rotation $\mathbf{q}_{k}^{(i)}$ by $\mathbf{u}_{p}^{(i)}=g_{1 / z}\left(\mathbf{q}_{k}^{(i)}{ }^{-1} \mathbf{z}_{p}^{(i)} \mathbf{q}_{k}^{(i)}\right)$. Accordingly the set of corrected FPs are calculated as

$$
\mathcal{U}^{(i)}=\left\{g_{1 / z}\left(\mathbf{q}_{k}^{(i)}{ }^{-1} \mathbf{z}_{p}^{(i)} \mathbf{q}_{k}^{(i)}\right): \mathbf{z}_{p}^{(i)} \in \mathcal{Z}^{(i)}\right\} .
$$

This transformation establishes apparent parallel views. However, (7) is dependent on the epipole point rather than on the point correspondences. For pure translation, the FPs fall on a line radiant from the epipole point. Conversely, the line intersecting two matched FPs ideally intersects the epipole point. Consequently, two such lines will intersect in the epipole point and each pair of FP matches will generate a measurement of the epipole point. Define the mapping $g_{L}:\left[\begin{array}{lll}x & y & z\end{array}\right] \mapsto\left[\begin{array}{lll}x & z & -y\end{array}\right]$. Then the line $\mathbf{l}_{p, q}^{(i)} \in \mathbb{R}^{3 \times 1}$, in homogeneous coordinates, intersecting the points $\mathbf{z}_{p}^{(c)}$ and $\mathbf{u}_{q}^{(i)}$, is $\mathbf{l}_{p, q}^{(i)}=g_{L}\left(\mathbf{z}_{p}^{(c)} \times \mathbf{u}_{q}^{(i)}\right)$ where $\times$ denotes the crossproduct. Accordingly, the set of all lines of matching pairs in the rotation unwrapped views is

$$
\mathcal{L}^{(i)}=\left\{g_{L}\left(\mathbf{z}_{p}^{(c)} \times \mathbf{u}_{q}^{(i)}\right):\{p, q\} \in \mathcal{M}^{(i)}\right\}
$$

In turn, the point at which two lines $\mathbf{l}_{r}^{(i)}$ and $\mathbf{l}_{s}^{(i)}$ intersect is $\mathbf{y}_{r, s}^{(i)}=g_{L}^{-1}\left(\mathbf{l}_{r}^{(i)} \times \mathbf{l}_{s}^{(i)}\right)$ where $r, s \in \mathcal{M}^{(i)}$ are 2-tuples and $g_{L}^{-1}:\left[\begin{array}{lll}x & y & z\end{array}\right] \mapsto\left[\begin{array}{lll}x & -z & y\end{array}\right]$. Accordingly, the set of all intersections of lines is

$$
\mathcal{Y}^{(i)}=\left\{g_{L}^{-1}\left(\mathbf{l}_{r}^{(i)} \times \mathbf{l}_{s}^{(i)}\right): \mathbf{l}_{r / s}^{(i)} \in \mathcal{L}^{(i)}, r \neq s\right\}
$$

Each member of the set $\mathcal{Y}^{(i)}$ is a measurement of the epipole $\mathbf{y}^{(i)}$ as of (7). Such sets of measurements are created for all views in $\mathcal{V}$.

In summary, the spatial information in the current view is condensed to a set of FPs $\mathcal{Z}^{(c)}$. From a finite visual memory $\mathcal{V}$, the featured point of the current image is matched by the FPs $\mathcal{Z}^{(i)}$ in the memory to create $\mathcal{M}^{(i)}$. Apparent parallel views are created by unwrapping the FPs creating $\mathcal{U}^{(i)}$. Based on these sets, lines intersecting the matched points are calculated giving $\mathcal{L}^{(i)}$. The intersections of all lines are calculated giving the epipole measurements in $\mathcal{Y}^{(i)}$. 


\section{E. Outlier rejection}

The FPs can reasonably be assumed to have small and well behaved deviations between views. However, the matching between points is not perfect and the epipoles are derived measurements and the calculations as such can be ill conditioned. Therefore, some measurement outlier rejection procedures are needed.

Poor matches can be detected by the relation (6). The fundamental matrix relative a view $(i)$ can be estimated by $\hat{\mathbf{F}}_{k}^{(i)}=\mathbf{A}^{\mathrm{T}}\left[\hat{\mathbf{p}}_{k}-\hat{\mathbf{p}}_{k}^{(i)}\right]_{\times} g_{\mathrm{R}}\left(\hat{\mathbf{q}}^{(i)}\right) \mathbf{A}^{-1}$, where $\hat{\mathbf{p}}_{k}$ and $\hat{\mathbf{p}}_{k}^{(i)}$ are calculated from (4) and (5) with the state estimates from (3). Then a match is rejected if $\mathbf{z}_{p}^{(i)^{\mathrm{T}}} \hat{\mathbf{F}}_{j}^{i} \mathbf{z}_{q}^{(j)}>\gamma_{F}$, where $\gamma_{F}$ is a geometric rejection threshold. This gives the outlier rejection

$$
\mathcal{M}^{(i)} \backslash\left\{\{p, q\} \in \mathcal{M}^{(i)}: \mathbf{z}_{p}^{(i)}{ }^{\mathrm{T}} \hat{\mathbf{F}}_{j}^{i} \mathbf{z}_{q}^{(j)}>\gamma_{F}\right\} \Rightarrow \mathcal{M}^{(i)}
$$

where $\backslash$ denotes the relative set complement or "set minus". The line derived from the matching point will be ill conditioned if the points lie too close together. Consequently point matches are rejected if $\left\|\mathbf{z}_{p}^{c}-\mathbf{u}_{q}^{(i)}\right\|<\gamma_{a}$, where $\gamma_{a}$ is a distance threshold. Accordingly

$$
\mathcal{M}^{(i)} \backslash\left\{\{p, q\} \in \mathcal{M}^{(i)} \forall\left\|\mathbf{z}_{p}^{c}-\mathbf{u}_{q}^{(i)}\right\|<\gamma_{a}\right\} \Rightarrow \mathcal{M}^{(i)} .
$$

If the lines are almost parallel, determining their intersection becomes ill conditioned. Therefore, an intersection is rejected if the angle between the lines is too small. Define the mapping $g_{l}:\left[\begin{array}{lll}x & y & z\end{array}\right] \mapsto\left[x /\left(x^{2}+y^{2}\right) \quad y /\left(x^{2}+y^{2}\right)\right]$. Then

$$
\mathcal{Y}^{(i)} \backslash\left\{g_{l}\left(\mathbf{l}_{r}^{(i)}\right) \cdot g_{l}\left(\mathbf{l}_{s}^{(i)}\right)<\gamma_{\times}: r, s \in \mathcal{M}^{(i)}\right\} \Rightarrow \mathcal{Y}^{(i)}
$$

where $\gamma_{\times}$is some parallelness threshold. Note that $g_{l}\left(\mathbf{l}_{r}^{(i)}\right)$. $g_{l}\left(\mathbf{l}_{s}^{(i)}\right)=\cos (\theta)$, where $\theta$ is the angle between the lines.

\section{COMPlementary MODELS}

In principle, the process models (1) and (2) could be combined with appended view positions $\mathbf{p}^{(i)}$ and the measurement model (7), giving a state space description of the system. However, propagating the statistics for $\mathbf{q}_{k}$ is difficult due to the constraint that $\mathbf{q}_{k} \in \mathbb{H}$. To overcome this, we use the customary approach of using complementary filtering with feedback. This means that the deviations of the INS state estimates (errors) from the true states are estimated instead of the states themselves. The feedback means that any non-zero deviation estimate is fed back, correcting the states propagated by (3). For a detailed treatment of complementary filtering for INS, see [10].

\section{A. Process model}

For complementary filtering, corresponding complementary models are needed. A first-order deviation model for (3) is

$$
\begin{aligned}
\delta \mathbf{p}_{k+1} & =\delta \mathbf{p}_{k}+d t_{k} \delta \mathbf{v}_{k} \\
\delta \mathbf{v}_{k+1} & =\delta \mathbf{v}_{k}+d t_{k}\left[\hat{\mathbf{R}}_{b, k}^{n} \tilde{\mathbf{f}}_{k}\right]_{\times} \delta \boldsymbol{\theta}_{k} \\
& +d t_{k} \hat{\mathbf{R}}_{b, k}^{n} \delta \mathbf{f}_{k}+d t_{k} \hat{\mathbf{R}}_{b, k}^{n} \mathbf{w}_{\mathbf{f}, k} \\
\delta \boldsymbol{\theta}_{k+1} & =\delta \boldsymbol{\theta}_{k}-d t_{k} \hat{\mathbf{R}}_{b, k}^{n} \delta \boldsymbol{\omega}_{k}-d t_{k} \hat{\mathbf{R}}_{b, k}^{n} \mathbf{w}_{\boldsymbol{\omega}, k} \\
\delta \mathbf{b}_{k+1} & =\delta \mathbf{b}_{k}+d t_{k} \mathbf{w}_{k}^{b},
\end{aligned}
$$

where $\delta \mathbf{p}_{k}, \delta \mathbf{v}_{k}, \delta \boldsymbol{\theta}_{k}$ and $\delta \mathbf{b}_{k}$ are the deviations of states of position, velocity, orientation, and sensor bias and $\mathbf{w}_{k}^{u}=$ $\left[\begin{array}{ll}\mathbf{w}_{\mathbf{f}, k}^{\mathrm{T}} & \mathbf{w}_{\boldsymbol{\omega}, k}^{\mathrm{T}}\end{array}\right]^{\mathrm{T}}$. Define the deviation state vector $\delta \mathbf{x}_{k}^{\text {ins }}=$ $\left[\begin{array}{llll}\delta \mathbf{p}_{k}^{\mathrm{T}} & \delta \mathbf{v}_{k}^{\mathrm{T}} & \boldsymbol{\theta}_{k}^{\mathrm{T}} & \delta \mathbf{b}_{k}^{\mathrm{T}}\end{array}\right]^{\mathrm{T}}$ and the corresponding process noise vector $\mathbf{w}_{k}^{\mathrm{T}}=\left[\begin{array}{ll}\mathbf{w}_{k}^{u \mathrm{~T}} & \mathbf{w}_{k}^{b \mathrm{~T}}\end{array}\right]^{\mathrm{T}}$. Then (9) can compactly be written in the form

$$
\delta \mathbf{x}_{k+1}^{\mathrm{ins}}=\mathbf{F}_{k}^{\mathrm{ins}} \delta \mathbf{x}_{k}^{\mathrm{ins}}+\mathbf{G}_{k}^{\mathrm{ins}} \mathbf{w}_{k} .
$$

The camera positions of old views $\hat{\mathbf{p}}^{(i)}$, given by $\hat{\mathbf{p}}_{k}, \hat{\mathbf{q}}_{k}$, and (4), obviously do not have any dynamics. Consequently, neither do the related deviations $\delta \mathbf{p}_{k}^{(i)}$ and therefore the process model is the identity $\delta \mathbf{p}_{k+1}^{(i)}=\mathbf{I}_{3} \delta \mathbf{p}_{k}^{(i)}$. When the image is captured, the error state is related by $\delta \mathbf{p}_{k}^{(i)}=\mathbf{T}_{k}^{\text {ins }} \delta \mathbf{x}_{k}^{\text {ins }}$, where $\left.\mathbf{T}_{k}^{\mathrm{ins}}=\left[\begin{array}{llll}\mathbf{I}_{3} & \mathbf{0}_{3 \times 3} & {\left[\hat{\mathbf{R}}_{b}^{n} \mathbf{p}_{c}^{b}\right.}\end{array}\right]_{\times} \mathbf{0}_{3 \times 6}.\right]$. Compounding the $P$ camera states $\delta \mathbf{x}^{\text {cam }}=\left[\begin{array}{lll}\delta \mathbf{p}^{(1)^{\mathrm{T}}} & \cdots & \delta \mathbf{p}^{(P)^{\mathrm{T}}}\end{array}\right]^{\mathrm{T}}$, we can write the IMU and camera position error processes jointly as

$$
\delta \mathbf{x}_{k+1}=\mathbf{F}_{k} \delta \mathbf{x}_{k}+\mathbf{G}_{k} \mathbf{w}_{k} \in \mathbb{R}^{15+3 P}
$$

where $\delta \mathbf{x}_{k}=\left[\begin{array}{ll}\delta \mathbf{x}_{k}^{\text {ins }} & \delta \mathbf{x}_{k}^{c a m}\end{array}\right]^{\mathrm{T}}, \mathbf{F}_{k}=\mathbf{F}_{k}^{\text {ins }} \oplus \mathbf{I}_{3 P}$ and $\mathbf{G}_{k}=$ $\left[\begin{array}{ll}\left(\mathbf{G}_{k}^{\text {ins }}\right)^{\mathrm{T}} & \mathbf{0}_{3 P \times 12}^{\mathrm{T}}\end{array}\right]^{\mathrm{T}}$. This gives a complementary process model for the whole system.

\section{B. Measurement model}

The dependence of the measurement model (7) of the system states comes in via $\Delta \mathbf{p}_{k}^{(i)}$. In terms of the deviation states

$$
\begin{aligned}
\Delta \mathbf{p}_{k}^{(i)}=\mathbf{d} \mathbf{q}_{k}\left(\hat{\mathbf{p}}^{(i)}-\right. & \delta \mathbf{p}^{(i)}-\hat{\mathbf{p}}_{k}+\delta \mathbf{p}_{k} \\
& \left.+\mathbf{C}\left(\delta \boldsymbol{\theta}_{k}\right) \hat{\mathbf{q}}_{k} \mathbf{p}_{(c)}^{b} \hat{\mathbf{q}}_{k}^{-1}\right) \mathbf{q}_{k}{ }^{-1} \mathbf{d}^{-1}
\end{aligned}
$$

where $\mathbf{C}\left(\delta \boldsymbol{\theta}_{k}\right) \approx \mathbf{I}_{3}-\left[\delta \boldsymbol{\theta}_{k}\right]_{\times}$is the direction-cosine matrix. Inserting (11) into (7) yields the complementary measurement model.

\section{State estimation}

The INS propagates state estimates according to (3) given inertial measurements $\tilde{\mathbf{u}}_{k}$. Based on these state estimates and the observed epipoles as of sets $\mathcal{Y}^{(i)}$, the deviation states $\delta \mathbf{x}_{k}$ shall be estimated. These are subsequently fed back to correct the INS and camera states.

In principle, all epipole measurements $\mathcal{Y}^{(i)}$ could be used as independent measurements. However, if the error covariances were identical up to scale, then equivalently we could use the weighted sample mean

$$
\tilde{\mathbf{y}}^{(i)}=\sum_{\mathbf{y}_{p}^{(i)} \in \mathcal{Y}^{(i)}} w_{p} g_{r}\left(\mathbf{y}_{p}^{(i)}\right),
$$

where $\sum w_{p}=1$, as a single measurement. This significantly reduces the computational burden of the measurement updates and lets us establish an estimated error covariance of the sample mean by

$$
\mathbf{C}^{(i)}=\sum_{\mathbf{y}_{p}^{(i)} \in \mathcal{Y}^{(i)}} w_{p}\left(\tilde{\mathbf{y}}^{(i)}-\mathbf{y}_{p}^{(i)}\right)\left(\tilde{\mathbf{y}}^{(i)}-\mathbf{y}_{p}^{(i)}\right)^{\mathrm{T}}+\lambda^{(i)} \mathbf{I}_{2} .
$$


where $\lambda^{(i)}=\frac{1}{M} \sigma_{y}, M$ is the number of elements in $\mathcal{Y}^{(i)}$, and $\sigma_{y}$ is a system parameter. The term $\mathbf{I}_{2} \lambda^{(i)}$ prevents the covariance from collapsing when only few measurements are available.

Stacking all $p \leq P$ available measurements at instant $k$ together into a measurement vector $\mathbf{y}_{k}$, combined with the process model (10), yields the complete state-space model,

$$
\begin{aligned}
\delta \mathbf{x}_{k+1} & =\mathbf{F}_{k} \delta \mathbf{x}_{k}+\mathbf{G}_{k} \mathbf{w}_{k} \\
\tilde{\mathbf{y}}_{k} & =\mathbf{h}_{k}\left(\hat{\mathbf{x}}_{k}, \delta \mathbf{x}_{k}\right)+\mathbf{v}_{k},
\end{aligned}
$$

where $\mathbf{h}_{k}(\cdot)$ represents the measurement model in (7), in terms of deviation states as of (11). The augmented noise vector $\mathbf{v}_{k}$ has a block diagonal covariance matrix $\mathbf{C}_{k}=\mathbf{C}^{(1)} \oplus \cdots \oplus$ $\mathbf{C}^{(p)} \in \mathbb{R}^{2 p}$.

The deviations $\delta \mathbf{x}$ are estimated by a square-root sigmapoint Kalman filter. The filter is summarized in Algorithm 1 and further explained below (for details see [11]). The necessary statistics are approximated by propagating "sigmapoints" through the measurement model. The sigma-points are generated from the square-root of the error covariance matrix $\mathbf{P}^{1 / 2}$ of the predicted deviations

$$
\delta \mathcal{X}_{\ell}= \begin{cases}0 & \ell=0 \\ \eta \cdot\left[\mathbf{P}^{1 / 2}\right]_{\ell} & \ell=1, \ldots, N \\ -\eta \cdot\left[\mathbf{P}^{1 / 2}\right]_{\ell-N} & \ell=N+1, \ldots, 2 N\end{cases}
$$

where $\left[\mathbf{P}^{1 / 2}\right]_{\ell}$ denotes the $\ell$ th column of $\mathbf{P}^{1 / 2}$. This is compactly expressed by the mapping $g_{\sigma}$. After the deviation is estimated, the error covariance matrix is updated by a series of rank-1 Cholesky downdates, denoted by $g_{\text {chol-d }}$. The time updates are performed by a standard square-root Kalman filter. The deviation process model (10) is linear and therefore the sigma-point transform is not needed to approximate the statistics. The mapping $g_{\mathrm{QR}}$ computes the time update of $\mathbf{P}_{k}^{1 / 2}$ using the QR factorization [12].

As a view $(i)$ is replaced in the memory, the corresponding state needs to be removed and the new one inserted. To keep the temporal order between states, the new view is added last in the state vector. The corresponding square-root covariance update is

$$
\mathbf{P}^{1 / 2}=\left[\begin{array}{c|c|c}
\mathbf{X} & \mathbf{0} & \mathbf{0} \\
\hline \cdot & \cdot & \mathbf{0} \\
\hline \mathbf{Y} & \mathbf{Z} & \mathbf{V}
\end{array}\right] \Rightarrow \mathbf{P}^{1 / 2}=\left[\begin{array}{ccc}
\mathbf{X} & \mathbf{0} & \mathbf{0} \\
\mathbf{Y} & \mathbf{W} & \mathbf{0} \\
\mathbf{T}^{\prime} \mathbf{X} & \mathbf{0} & \mathbf{0}
\end{array}\right]
$$

where $\mathbf{X}$ and $\mathbf{V}$ are lower triangular matrices, the blank rows correspond to the rows related to the removed view state, $\mathbf{W}$ is the lower-triangular Cholesky factorization of $\mathbf{Z} \mathbf{Z}^{T}+\mathbf{V} \mathbf{V}^{T}$, and $\mathbf{T}^{\prime}=\left[\begin{array}{ll}\mathbf{T}^{\text {ins }} & \mathbf{0}_{3 \times 3(i-1)}\end{array}\right]$.

\section{ADAPTIVE VISUAL MEMORY}

The visual memory $\mathcal{V}$ has finite size $P$ but new images arrive at regular intervals. Therefore, old images need to be removed from the buffer at some point. Unfortunately, due to the realtime constraint, the size $P$ is restricted and therefore images might have to be discarded while still contributing to the navigation solution. However, discarding the oldest view

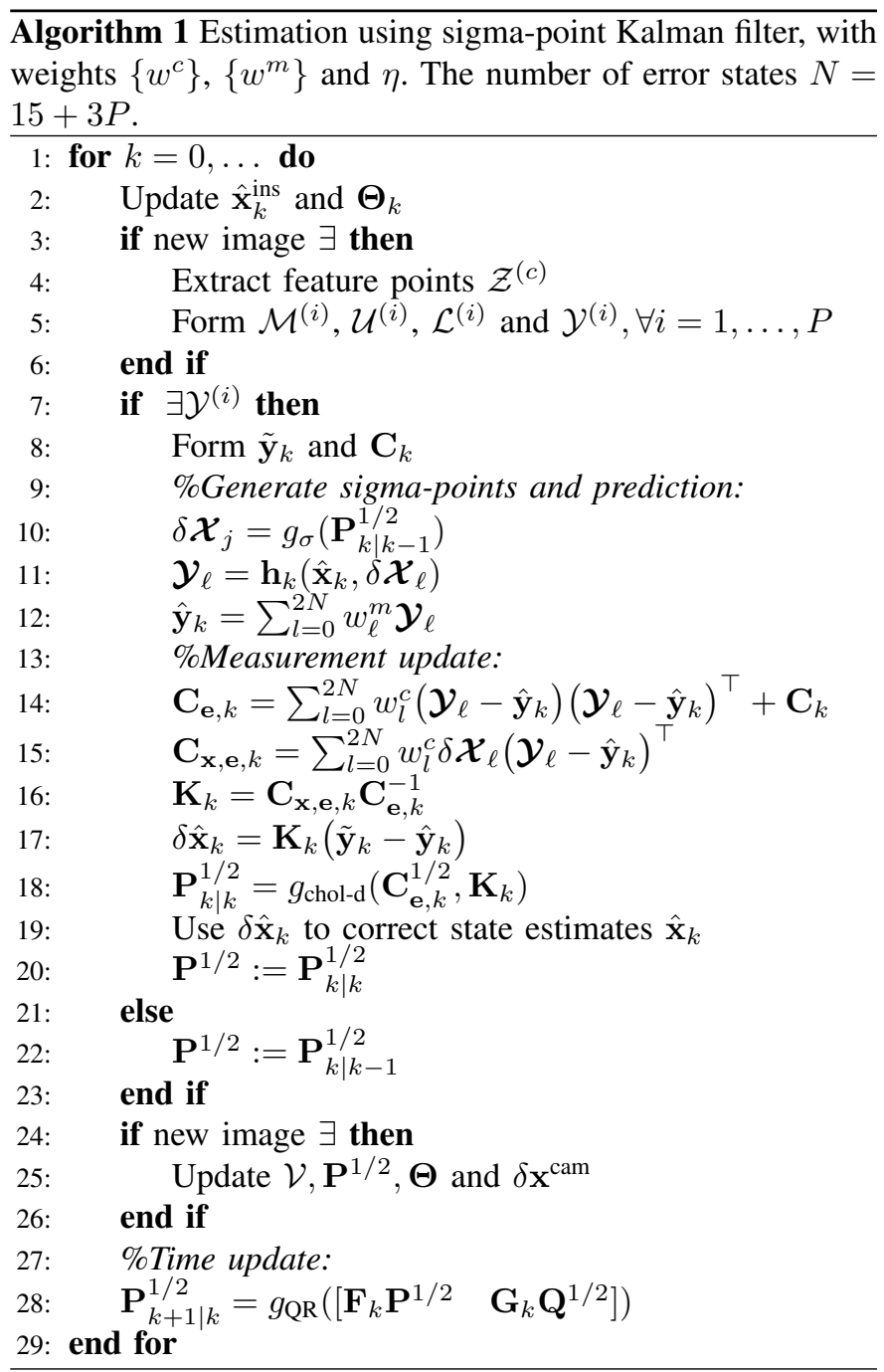

is in general not a good strategy. On the one hand, the oldest image will have the largest time baseline and as such the greatest potential value. On the other hand, the oldest view is also the one that is the most likely to have no perspective overlap with the current and future views, in which case it is of no value. Consequently, a utility measure of each stored view $(i)$ is needed.

The value of a view is naturally measured by its statistical coupling to the current and future navigation deviation states $\delta \mathbf{x}_{k}^{\text {ins }}$. The second-order statistics of this coupling will be determined by the covariance of the epipole measurements, the geometry between views, and the covariance of the deviation states. For each new view, the coupling to the current deviation state can be defined by the corresponding Kalman gain submatrix (see Algorithm (1)) $\overline{\mathbf{K}}_{k}=\mathbf{K}_{k(1: 15, \cdot)}$. By dividing the gain submatrix into components related to each individual view, $\overline{\mathbf{K}}_{k}=\left[\mathbf{K}_{k}^{(1)} \cdots \mathbf{K}_{k}^{(p)}\right]$, the contribution of each view $\mathbf{K}_{k}^{(i)} \in \mathbb{R}^{15 \times 2}$ is separated in the sense that $\delta \hat{\mathbf{x}}_{k \mid k}^{\text {ins }}=\sum_{i=1}^{p} \mathbf{K}_{k}^{(i)} \mathbf{e}_{k}^{(i)}$, where $\mathbf{e}_{k}^{(i)}$ is the innovation of the visual constraint of the $(i)$ th view.

The different states have different units and might be valued 
differently. Therefore, a weight vector $\mathbf{w}_{\mu} \in \mathbb{R}^{15 \times 1}$ weighing the different states is necessary. Define an instantaneous coupling factor

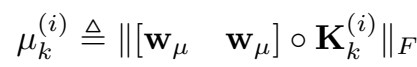

where $\|\cdot\|_{F}$ is the Frobenius norm and $\circ$ is the Hadamard product. If there is no observation related to the view $(i)$ at time $k, \mu_{k}^{(i)}=0$. Since $\left\|\mathbf{w}_{\mu} \circ \delta \mathbf{x}_{k \mid k}^{\text {ins }}\right\|_{2} \leq \sum_{i=1}^{p} \mu^{(i)}\left\|\mathbf{e}_{k}^{(i)}\right\|_{2}$, $\mu_{i}$ quantifies the potential gain each view $(i)$ has in correcting the navigation solution. For simplicity and motivated by the strong coupling of all other states to the position state, we have typically used $\mathbf{w}_{\mu}=\left[\begin{array}{ll}\mathbf{1}_{3 \times 1} & \mathbf{0}_{12 \times 1}\end{array}\right]^{\mathrm{T}}$.

The instantaneous coupling factor $\mu_{k}^{(i)}$ gives a measure of the coupling of view $(i)$ with the current states $\delta \mathbf{x}_{k}^{\text {ins }}$. Also, due to the dead-reckoning nature of the system, it gives an indication of the coupling to future states $\delta \mathbf{x}_{k}^{\text {ins }}$. However, due to the risk of non-overlapping scenes of the views, without any further information about the future trajectory, the further away in the future the less information the current coupling factor $\mu_{k}^{(i)}$ carries about future coupling factors. Consequently, to determine the utility of a view, we need to balance instantaneous coupling factors of the current and past views. For this reason, we define a view utility $g_{k}^{(i)}$ for each camera sample $k$ based on a history of instantaneous coupling factors, computed as

$$
g_{k}^{(i)}=\lambda g_{k-1}^{(i)}+\mu_{k}^{(i)},
$$

where $0 \leq \lambda<1$. The view utilities $g_{k}^{(i)}$ are kept track of for each view in the visual memory. At the end of each measurement update, the view $(j)=\arg \min _{(i)}\left(g_{k}^{(i)}\right)$ with the smallest utility is removed from the buffer and the current view $(c)$ is inserted in its place. A nonzero initial frame utility $g_{0}$ can be used to favor new images. This will be useful if large memory sizes are used and $\lambda$ is small. The decaying memory in $g_{k}^{(i)}$ makes it possible for "good" views to survive in the memory during periods of motion blur or look-around motions. Since there is nothing epipole specific to the adaptive visual memory, the utility measure could be used for any finite memory, e.g. for positions of individual feature points.

\section{TIME SYNCHRONIZATION AND TEMPORAL ORDERING}

No synchronization of the sensors is assumed. For realtime processing, this implies that the time instants of the measurements $t_{k}$ and the time indices $k$ need to be estimated recursively. The sensors are assumed free running providing data at regular intervals with respect to their local oscillators. This means that the dynamic of the time instants relative the processing unit clock can be modeled by [7]

$$
\left[\begin{array}{c}
t_{\ell} \\
\alpha_{\ell}^{-1}
\end{array}\right]=\left[\begin{array}{cc}
1 & d_{\ell} \\
0 & 1
\end{array}\right]\left[\begin{array}{c}
t_{\ell-1} \\
\alpha_{\ell-1}^{-1}
\end{array}\right]+\left[\begin{array}{c}
0 \\
w_{\ell}
\end{array}\right]
$$

where $t_{\ell}$ is the time instant of the $\ell$ th measurement from a peripheral unit with respect to the central clock; $\alpha_{\ell}^{-1}$ is the inverse of the clock pace of the peripheral oscillator; $d_{\ell}$ is the period of the measurement with respect to the peripheral clock; and $w_{l}$ is some random frequency drift in the oscillator.
In turn, the time stamps from the central unit can be modeled by

$$
\gamma_{\ell}=t_{\ell}+\tau+v_{\ell}
$$

where $\tau$ is a constant mean communication delay and $v_{\ell}$ is a random delay component. Relations (14) and (15) make up a timing state-space model for the sensor units. The state and consequently the measurement time instants $t_{\ell}$ can be estimated by a Kalman filter giving a time synchronization between the asynchronous measurements. The estimates $\hat{t}_{\ell}$ in turn give the timing indices $k$ and the time differentials $d t_{k}$ needed for all filter time updates.

Due to varying communication delays and thread and buss scheduling, it cannot be assumed that measurements will arrive in the order of their estimated sampling instants $\hat{t}_{\ell}$. To order the measurements in realtime, predictive temporal ordering is used [7]. Based on the timing estimates and the state space model (14), the timing of the next measurement is continually predicted by

$$
\hat{t}_{\ell+1 \mid \ell}=\hat{t}_{\ell \mid \ell}+d_{\ell} \hat{\alpha}_{\ell \mid \ell}^{-1} .
$$

The updated timing estimate of an incoming measurement is compared with the predicted timing of the next time measurement of the other sensor. If it is within four standard deviations of the predicted timing estimate, the system waits for the measurement of the other sensor to arrive to determine which temporal order they should have. Otherwise, the system proceeds and processes the incoming estimate. If a measurement is incorrectly ordered as judged by the updated timing estimates, the measurement is rejected. In the considered setup, this happens in less than 1 in $10^{5}$ measurements.

\section{REALTIME IMPLEMENTATION}

The visual-aided INS described in Sections II-VII has been implemented in realtime. The implementation runs on standard laptops with off-the-shelf sensor equipment. All code has been written in $\mathrm{C} / \mathrm{C}++$.

\section{A. Hardware}

The required hardware is an IMU, a camera, and a processing platform. Various MicroStrain IMUs (GX2) have been used with the system. The camera is an AVT Guppy WVGA F-036B with 8-bit grayscale images and $752 \times 460$ pixels resolution. The camera features a FireWire (IEEE1394) interface. Several standard laptops, all running Linux and with Intel i5 and i7 CPUs and 2-6 GB RAM, have successfully been used as processing platforms. Figure 2 shows the sensor units.

\section{B. Processing framework}

The foundation for the system software implementation is an in-house developed soft-realtime processing framework. The framework contains a set of virtual classes that define internal interfaces and threading functionality and declare main runtime routines. Main filtering components, i.e. the blocks as of Fig. 1, inherit these virtual classes and therefore have to define the virtual runtime routines. This way, conformity of the framework-derived components is ensured. 


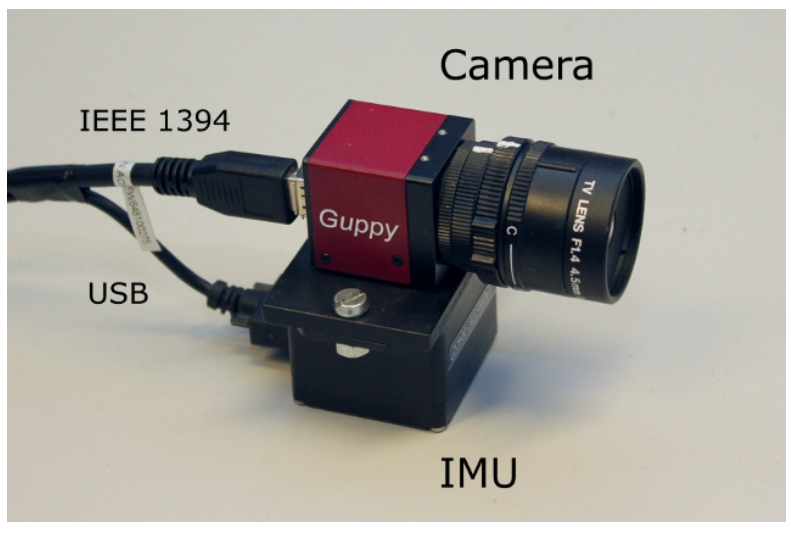

Fig. 2. Sensor units of the visual-aided INS implementation. The camera is rigidly mounted together with the IMU. Each sensor unit is connected to the processing platform with asynchronous data busses, i.e. IEEE-1394 and USB.

Components for controlling and receiving and parsing data from each sensor and rendering the filtering results have been developed for the processing framework. Control and reception of the data from the IMU is based on standard POSIX (IEEE 1003) system calls. Control and reception of the data from the camera is based on the IIDC 1394-based Digital Camera Specifications high level programming interface library libdc1394. The rendering is based on the Simple DirectMedia Layer (SDL) library.

The processing framework uses different threads for receiving data from each sensor, performing the filtering, and rendering the result. The threading has been implemented with POSIX threads (pthreads). The different framework components are connected to each other by callback functions. The different threads hand over data using internal circular buffers.

\section{Feature-point methods}

For the FP detection, description, and matching, as described in Section III, the SURF method was used. The SURF method was selected, despite its weaknesses and lack of theoretical foundation, due to its low computational cost. However, available SURF implementations were found too slow and instead an in-house developed implementation of SURF with some minor modifications was used. The implementation is capable of processing a WVGA image in $\sim 30[\mathrm{~ms}]$ on a standard laptop. To bound the computational cost for the image processing, to ensure realtime capability, an upper limit $N_{\max }$ was set on the number of FPs. The FPs were selected based on the distinctiveness (detection) measure, i.e. the determinant of the Hessian.

Recently, multiple improved FP methods have been presented, e.g. [13]. They should be favored if the system is reimplemented.

\section{Filtering}

The filtering as described in Algorithm 1 mainly consists of matrix operations and control sequences. The matrix operations has been implemented using the library ITPP (including the BLAS and LAPACK libraries) and LINPACK for the Cholesky downdating.

\section{Computational COMPLEXITY AND COST}

Naturally, the computational cost relative to the computational capability of the processing platform determines the possibility of running a filter in realtime. However, many of the processing stages have parameters affecting the computational cost and at the same time, the system performance; meanwhile, the computational capability varies with different platforms. Therefore, the scaling of the computational cost becomes important, since it enables a trade-off between the computational cost, the performance, and the computational platform.

As Fig. 1 illustrates, the system can be divided into a set of processing blocks. Naturally, the computational cost divides in the same blocks. Based on an inspection of the related processing, the INS, the synchronization and temporal ordering, and the visual memory is directly seen to have marginal computational costs and is therefore omitted from this discussion. The remaining blocks from Figure 1 are the FP extraction and matching, the epipole calculation, and the complementary filtering.

The FP extraction and matching consists of an image processing part, a FP description part, and the matching. The image processing cost scales linearly with the number of pixels $N_{\text {pix }}$, the FP description cost scales linearly with the number of FPs $N_{\mathrm{FP}}$, and the matching cost scales with the product of the number of FPs in each set that is matched. All these calculations have to be done at an update rate of $f_{\text {cam }}$. This gives the computational cost

$$
c_{\mathrm{FP}}=\left(c_{\mathrm{pix}} \cdot N_{\mathrm{pix}}+c_{\eta} \cdot N_{\mathrm{FP}}+c_{\mathrm{M}}\left(P N_{\mathrm{FP}}\right) \cdot N_{\mathrm{FP}}\right) f_{\mathrm{cam}}
$$

where $c_{\text {pix }}$ is the computational cost per pixel, $c_{\eta}$ is the cost for creating a descriptor for each FP, and $c_{\mathrm{M}}$ is the cost of a single match, and it has been assumed that the number of FPs $\left(N_{\mathrm{FP}}\right)$ is roughly the same in all sets. A more detailed analysis can be found in [14]. Epipole calculation entails calculating the sets $\mathcal{U}^{(i)}, \mathcal{L}^{(i)}$, and $\mathcal{Y}^{(i)}$. The calculation of $\mathcal{Y}^{(i)}$ scales with the square of the number of matches and is therefore dominant. The calculations have to be done for every new view and for each view in $\mathcal{V}$. However, only a fraction of all FPs give matches and only a few arithmetic operations are required to calculate the cross product. Therefore, the computational cost of the epipole calculation is marginal in comparison to the matching, i.e. the last term in (17). The complementary filtering requires two types of updates: the time updates, which are done for every inertial measurement; and the measurement updates, which are done for every view. Since the filter is run in a complementary mode with feedback, the time update only entails updating the covariance matrix. This entails a QR-decomposition that scales as the cube of the state vector dimension. The measurement update scales similarly with the dimension of the measurements. However, the camera update rate is typically much lower than the IMU update rate and 
therefore the cost is marginal. This gives the computational cost

$$
c_{\text {filter }}=c_{\text {tud }} \cdot(3 P+15)^{3} \cdot f_{\text {ins }} .
$$

where $c_{t u d}$ is the time update cost per covariance matrix element and $(3 P+15)$ is the state dimension of the complementary filter.

Naturally, the exact values of the coefficient $c_{\text {pix }}, c_{\eta}, c_{\mathrm{M}}$, and $c_{\text {tud }}$ depend on the filtering implementation and processing platform. However, to make a qualitative analysis of the computational cost, only rough values are needed. Based on runtime measurements with Callgrind (Valgrind profiling tool), on the laptops in use, the following rough values of the coefficient (in [s]) were observed

$c_{\text {pix }} \sim 10^{-7}, \quad c_{\eta} \sim 10^{-4}, \quad c_{\mathrm{M}} \sim 10^{-7}, \quad$ and $\quad c_{t u d} \sim 10^{-9}$.

These values can directly be seen to be reasonable considering the number of arithmetic operations involved in the different processing stages. Assume $f_{\text {ins }}=250[\mathrm{~Hz}]$ and $f_{\text {cam }}=10[\mathrm{~Hz}]$. Based on these values, we may note that

1) For small number of feature points $\left(N_{\mathrm{FP}} \lesssim 100\right)$ and small memory sizes $(P \lesssim 30)$, the computational cost is dominated by the basic image processing.

2) For larger number of feature points but small memory sizes $(P \lesssim 30)$, the computational cost is dominated by the matching.

3) For larger memory sizes, the cost is dominated by the filter time update.

This complexity analysis is rather general. If the factor $\left(P N_{\mathrm{FP}}\right)$ is exchanged against the general number of FPs, that the FPs of the current image is matched against, and $(3 P+15)^{3}$ is changed to some arbitrary dimension of the filter state, then the analysis applies to any Kalman-based filtering of imagery data based on FPs.

The major trade-off between parameters, performance, and computational cost becomes that between the memory size $P$ and the maximum number of FPs. We have generally used a trade-off around 200 FPs and $P=10$. With these settings, the feature point extraction and matching make up roughly $80 \%$ of the computational cost and the filter time-update roughly $20 \%$.

\section{EXPERIMENTAL AND RESUlts}

An experiment was conducted during which we walked around the laboratory. The system was held in the hand facing forward in the walking direction. The system was moved around in a figure of an eight followed by three laps around the "lower" circle in the eight. The trajectory is shown in Fig. 3.

The trajectory was around $200[\mathrm{~m}]$. Obviously, the heading estimate drifts away after some time but since the trajectory is closed-loop, many of the induced errors cancel out and the estimate of the end position estimate is only about $2[\mathrm{~m}]$ off from the starting position.

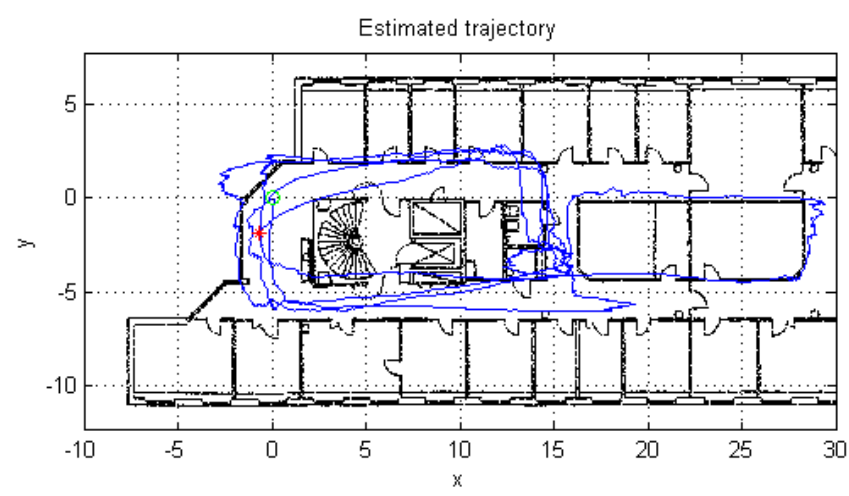

Fig. 3. Example trajectory estimated by the visual-aided INS plotted on a map overlay. A green circle indicates the starting position while a red cross indicates the final position.

\section{CONCLUSION}

We have presented a realtime visual-aided INS. The system is capable of running on a standard laptop and only requires off-the-shelf hardware. A closed-loop trajectory shows an error around $1 \%$ of the traveled distance.

\section{REFERENCES}

[1] A. I. Mourikis and S. I. Roumeliotis, "A multi-state constraint Kalman filter for vision-aided inertial navigation," in Proceedings of the IEEE International Conference on Robotics and Automation, (Rome, Italy), pp. 3565-3572, April 2007.

[2] M. Veth, Fusion of imaging and inertial sensors for navigation. $\mathrm{PhD}$ thesis, Air Force Institute of Technology, 2006.

[3] P. Pinies, T. Lupton, S. Sukkarieh, and J. Tardos, "Inertial aiding of inverse depth slam using a monocular camera," in Robotics and Automation, 2007 IEEE International Conference on, pp. 2797 -2802, April 2007.

[4] J. Fletcher, M. Veth, and J. Raquet, "Real-time fusion of image and inertial sensors for navigation," tech. rep., Air Force Institute of Technology, 2007.

[5] D. Zachariah and M. Jansson, "Camera-aided inertial navigation using epipolar points," in Proc. PLANS2010, 2010.

[6] D. Diel, P. DeBitetto, and S. Teller, "Epipolar constraints for visionaided inertial navigation," in Application of Computer Vision, 2005. WACV/MOTIONS '05 Volume 1. Seventh IEEE Workshops on, vol. 2, pp. $221-228$, Jan. 2005.

[7] J.-O. Nilsson and P. Händel, "Time synchronization and temporal ordering of asynchronous sensor measurements of a multi-sensor navigation system," in Proc. PLANS2010, 2010.

[8] C. Jekeli, Inertial Navigation Systems with Geodetic Applications. de Gruyter, 2001.

[9] R. Hartley and A. Zisserman, Multiple View Geometry in Computet Vision. Cambridge University Press, 2000.

[10] J. A. Farrell, Aided Navigation. Mc Graw Hill, 2008.

[11] R. Van der Merwe and E. Wan, "The square-root unscented Kalman filter for state and parameter-estimation," in Proc. ICASSP01, vol. 6, 2001.

[12] T. Kailath, A. Sayed, and B. Hassibi, Linear Estimation. Prentice Hall, 2000.

[13] M. Calonder, V. Lepetit, C. Strecha, and P. Fua, "BRIEF: Binary Robust Independent Elementary Features," in European Conference on Computer Vision, Sept. 2010.

[14] J. Kristensen, "Implementering och karakterisering av punktdeskriptorer för realtidsbehandling av fotodata för kamerastödd tröghetsnavigering," Master's thesis, KTH Royal Institute of Technology, 2012. (in Swedish). 